\title{
A Trend Between Cold Debris Disk Temperature and Stellar Type: Implications for the Formation and Evolution of Wide-Orbit Planets
}

\author{
Nicholas P. Ballering ${ }^{1}$, George H. Rieke ${ }^{1}$, \\ Kate Y. L. Su ${ }^{1}$ and Edward Montiel ${ }^{1,2}$ \\ ${ }^{1}$ Steward Observatory, University of Arizona, Tucson, AZ, USA \\ ${ }^{2}$ Department of Physics \& Astronomy, Louisiana State University, Baton Rouge, LA, USA
}

\begin{abstract}
Cold debris disks have the potential to answer many outstanding questions in wideorbit planet formation and evolution. We characterized the infrared excess SEDs of 174 cold debris disks with Spitzer IRS and MIPS. We found a trend between the temperature of the disks and the stellar type of the stars they orbit. This argues against the importance of strictly temperature-dependent processes (e.g. ice lines) in setting the dimensions of cold debris disks. We also found no evidence that delayed stirring causes the trend. The trend may result from outward planet migration that traces the extent of the primordial protoplanetary disk, or from planet formation that halts at an orbital radius limited by the efficiency of core accretion. For the full details of this work, see Ballering et al. (2013).
\end{abstract}

Keywords. circumstellar matter - infrared: stars - interplanetary medium

\section{Introduction}

Detecting wide-orbit planets is difficult, but we can study their formation and evolution from observations of cold debris disks because planets may sculpt sharp inner edges in the disks. The location of a cold disk may trace the extent of outward planet migration or the limits of efficient planet formation. These effects predict that cold debris disks around early type stars will be warmer than those around later type stars. Alternatively, ice lines in the protoplanetary disk phase could set the location of debris disks by creating a dust trap. The location of warm debris disks is thought to be set by the water ice line (Morales et al. 2011), and cold debris disks could be set by the ice line of other species. This effect predicts that cold debris disks will have similar temperatures around all stellar types.

\section{Methods and Results}

We analyzed the SEDs of 546 main sequence stars with Spitzer IRS and MIPS data. To obtain each debris disk temperature(s), we fit the excess SED with one or two blackbody curves. Our definition of a "cold" debris disk was the coldest component below $130 \mathrm{~K}$. We found 51 single component warm disks, 100 single component cold disks, and 74 two component disks. For 25 very cold disks, the lack of data at wavelengths longer than $70 \mu \mathrm{m}$ prevented us from accurately measuring their temperatures, so we assigned them upper limit temperatures of $45 \mathrm{~K}$. By plotting the cold disk temperatures vs. stellar temperatures, we see that cold disks tend to be warmer around early type stars (see Fig. 1). Could age play a confounding role in this relation? Because early type stars are preferentially younger than late type stars, this trend would arise if debris disks cool as 


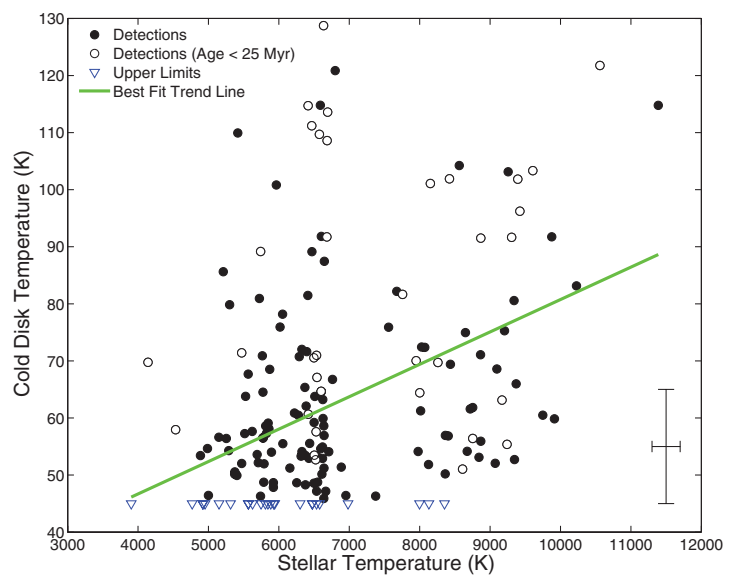

Figure 1. The temperature of the cold disk component versus the temperature of the disk's host star. Open circles are young systems (where active planet formation may still be occurring) and triangles are upper limits. Although there is substantial scatter in the cold component temperatures (reflecting the degree of variability among debris disk properties), a correlation between cold debris disk temperature and stellar type is evident. Note, for example, that there are no disks colder than $50 \mathrm{~K}$ around stars hotter than $8500 \mathrm{~K}$, in comparison with the large number of disks with temperature $<45 \mathrm{~K}$ around cooler stars. The line is the best fit to the data, determined by a Bayesian linear regression, $T_{\text {cold }}=0.00568 \times T_{\star}+24.0$. Thus, the trend is significant at a level greater than $4.5 \sigma$. A representative error bar is in the lower right.

they age. Such behavior is predicted by delayed stirring models, wherein an annulus of active dust production propagates outward through a continuous disk (Wyatt 2008). We saw no evidence for cold debris disks around the same stellar type cooling with age after the first few tens of Myr.

\section{Discussion and Conclusions}

What determines the locations of cold debris disks? This trend suggests that their locations are not set by a temperature-dependent process, such as an ice line. Perhaps their locations are set by planets migrating outwards via scattering with planetesimals. Migration will stop towards the outer edge of the disk where the surface density of planetesimals drops. From observations of protoplanetary disks, $R_{\text {disk }} \propto M_{*}^{0.63}$ (Scholz et al. 2006; Andrews et al. 2010), which predicts $T_{\text {disk }} \propto T_{\star}^{1.38}$, consistent with the observed trend. Another possibility is that the inner edge of a cold debris disk represents the radial limits of efficient planet formation. The timescale for core accretion scales as $t \propto R^{3} M_{\star}^{-3 / 2}$ (Kenyon \& Bromley 2008). The inner edge of the cold disk will be set at the location where $t$ reaches a given threshold, so setting $t$ constant yields $R_{\text {disk }} \propto M_{\star}^{1 / 2}$. This translates to $T_{\text {disk }} \propto T_{\star}^{3 / 2}$, which is also consistent with the observed trend.

\section{References}

Andrews, S. M., Wilner, D. J., Hughes, A. M., Qi, C., \& Dullemond, C. P. 2010, ApJ, 723, 1241

Ballering, N. P., Rieke, G. H., Su, K. Y. L., \& Montiel, E. J. 2013, ApJ, 775, 55

Kenyon, S. J., \& Bromley, B. C. 2008, ApJS, 179, 451

Morales, F. Y., Rieke, G. H., Werner, M. W., Bryden, G., Stapelfeldt, K. R., \& Su, K. Y. L. 2011, ApJ, 730, L29

Scholz, A., Jayawardhana, R., \& Wood, K. 2006, ApJ, 645, 1498

Wyatt, M. C. 2008, ARA\&A, 46, 339 